\title{
Integration of Internet of things and Artificial Neural Networks for Efficient Mechanism
}

\author{
Apoorv Saxena \\ SRM University \\ Kancheepuram District, \\ Near Potheri Railway Station, Chennai, India
}

\begin{abstract}
Internet of things and Artificial Neural Network are two diverse types of technologies. Their growth in last decade is astonishing and are in people's reach. However, their integration together will result in elevated performance in field which are inevitable to touch right now like space technologies and many safety issues in industries globally. there are over 50 billion connected devices in IoT until the end of the next decade. This huge data has patterns in it which is difficult for humans to deal with as it could be structured as well as unstructured, Artificial Neural Networks can deal with this data and can learn from the pattern present in the data from which they can tell which Protocol/Steps to follow in different Circumstances. Loses of any type could be Minimized as well as productivity can be enhanced. IoT and ANN can be integrated by using different types of technologies like, Eclipse Mosquito and Eclipse Paho ,Mqtt, different types of IoT sensor and other component for ANN like heterogeneous local device processors, decompose monolithic deep model network architectures etc. this system if implemented on massive scale can bring tremendous change in ways we construct our safety policy and backup plans as everything can be done on real time. Thus in this paper we focus our attention on integration of ANN and IoT For purpose like Safety.
\end{abstract}

\section{Keywords}

Artificial Neural Networks, IoT , Safety Mechanism

\section{INTRODUCTION}

The Internet of things is regarded as the next technology wave after the Internet and seems to be bigger then that from a report it is estimated that it's potential is around 11 trillion \$. The Internet of things is based on Intelligent and SelfConfiguring nodes(things) interconnected in a dynamic and global network infrastructure. it represents one of the most pervasive computing scenario.

Thus it connect everything for sharing information and communicating and performing some action thorough information sensing devices known as sensor with agreed protocol since its inception growth of IoT has being exceptional because of its vast uses and economic benefits only with 12 sensor of diverse types we can create 32,000 types of sensor applications. However any Industrial Scale system is hard to manage because as complexity overall extends the human intellectual capacity as most of the data are in the form of unstructured data. It is hard to reach any conclusion with such a huge data and error possibilities also increases. On the Other hand Artificial Neural Networks(ANN) learn from the Input data given to them and make probability and help to figure out different condition in which we can act according to the data of the sensor thus with time this system become more efficient and analyses itself.
Thus this Technology remains promising in the area which generate a lot of data and could be a breakthrough to many Artificial Neural Networks is a series of fully-connected layers collectively form a ANN architecture with each layer comprised by a collection of units (or nodes). Training data set (e.g., audio, images) initialize the values of the first layer (the input layer). The output layer (the last layer) corresponds to inference classes, with units capturing individual inference categories (e.g., music or cat). Hidden layers are contained between input and output layers. Collectively, they are responsible for transforming the state of the input layer into the inference classes captured in the last layer. Every unit contains an activation function that determines how to calculate the unit's own state based on units from the immediately previous layer. The degree of influence of units between layers vary on a pairwise basis determined by a weight value. Naturally, the output of the unit also helps to determine the unit state in the next layer. Training Data Set are provided by the IoT sensor fixed on the system and are able to contact each other This real time phenomena is performed by using MQTT(message query telemetry transport) integrated with Eclipse Mosquitto and Eclipse Paho ,MQTT is a client server architecture. Here is a broker and a client. Broker act like a server and it take query from thousands of client(sensor in our case and process it) it is highly efficient because of it's real time property and less code foot print with low battery usage ,support In low network with eclipse paho and where Eclipse paho at an end will subscribe the data while at the other end eclipse Mosquitto while publishing the data. If our system finds any irregularity or failure it can compare with the previous data and action could be taken as per the situation in old system we follow very limited steps for security/Safety it cannot work in any circumstances example War Tanks are vulnerable to airstrike but by this IoT can sense the presence and ANN can find the most preferred way to be safe which with human accuracy is not so efficient. There are enormous field and industries were this system could be used ex-Toxic Detection and Remedy, Sequence Generation , Mitosis Detection , Grading Essays , Crude and other industries for the safety measure.

\section{ARTIFICIAL NEURAL NETWORKS AND INTERNET OF THINGS: THE NEED FOR THEIR INTEGRATION}

The two worlds of ANN and IoT have seen an independent evolution. However, several mutual advantages deriving from their integration have been identified in literature and are foreseen in the future on the one hand IoT is able to collect large amount of data while ANN can learn from this data and draw a conclusion. ties. IoT is characterized by a very high heterogeneity of devices, technologies, and protocols. Therefore, scalability, interoperability, reliability, efficiency, availability this problem can easily be tackled by the usage of 
ANN. Following are some reason that these technologies need to be integrated.

Training Data Set-Training data set is very important as ANN draw output from this Data set only. This Data set is provided by IoT it means large amount of information systems, huge amount of structured and non-structured data. Thus, it gives Data to ANN also IoT gives an unprecedented advantage we can make several combinations through sensor for performing distinct functions also powerful security algorithm will be involved because of the huge Training set making ANN better.

Realizing potential also requires as to address huge challenges like Security Concern about location and other sensitive being located and transmitted, There is a lack of industry standard which is a challenge for large scale implementation due to high number of sensor and other material cost can initially increase but in long run it will be more effective as data could also be monetized IoT and ANN faces different types of Challenges that could be divided into five types

\section{Architectural Challenges \\ 2. Technical Challenges \\ 3. Hardware Challenges \\ 4. Private and Security Challenge \\ 5. Standard Challenges}

These challenges will be viewed in this paper and how they can be overcome also for some it is quite difficult to justify this as industries is growing in a rapid speed but with decreasing cost of the hardware and other component it has become economic feasible.

\section{TRANING ARTIFICIAL NEURAL NETWORKS WITH INTERNET OF THINGS}

ANN is a feed-forward, artificial neural network that has more than one layer of hidden units between its inputs and its outputs and input layers are less than the output layers usually. A simple example could be linear Classifier given Below.

$\mathrm{w} \cdot \mathrm{x}+\mathrm{b}=\mathrm{y}$

This take the input that could be structured and unstructured data and apply above linear function to generate its predictions this linear function is just a giant matrix multiply. All the Input is denoted as vector X. W stands for weight and B for Bias weight are like different outcome. When we get the score $\mathrm{Y}$ we convert it into probability for converting Score into Probability we use SoftMax function.

$\mathrm{S}\left(\mathrm{y}_{\mathrm{i}}\right)=\frac{e^{y_{i}}}{\sum_{j} e_{y_{j}}}$

This can take any type of score and turn in to probability SoftMax function we can state with reduce in inputs the linear classifier can become unsure as output could be of similar probabilities thus more training set is always considered.

There is a better way of finding probabilities by using cross entropy

$D(S, L)=-\sum_{i} L^{i} \log \left(S^{i}\right)$

This either convert $d$ (Distance) into Zero or one as $\mathrm{S}$ came from SoftMax function which can be zero or one making convinent for machine to figure out which is best outcome also the loss could be reduced as most of the training set will give zero output in distance.

This loss can be reduced by

$$
L=\frac{1}{N} \sum_{I} D\left(S, L_{i}\right)
$$

This will give you a humongous sum as in SoftMax function "W" is a huge matrix and then it gets sum by sigma thus to minimize it we use the above equation.

This system requires a great Computation power which is possible to attain with current technologies by using GPUs and other components there are other tool which are used for Maximizing hardware usage but it still takes a lot of testing time and with increased in training data set because of IoT we need more computation power and resources that a hurdle to implement this system.

Internet of things can be implemented by ANN just by passing the right data set Consider a Railway Locomotive where we could fix sensor on engine, brake, horn, gates and other places all this sensor are integrated to each other and extract information from the system this information is then stored in the common node or on cloud from where they can be sent to ANN Computation area and if the system record anything unusual they can sent back the data to the operator or sensor and best possible option can be taken correctly. There are many protocol and technologies that should be follow so the system could work on unfavorable condition. For this MQTT is preferred it's an extremely lightweight messaging protocol. Its publish/subscribe architecture is designed to be open and lucid to implement. Single MQTT server can support up to thousands of remote clients. These characteristics make MQTT ideal for use in constrained environments where network bandwidth is low or remote devices that might have limited processing capabilities and memory, need to be supported. The MQTT protocol is based on publish/subscribe model. Publishers can send the messages to the topics, configured on the MQTT server (also called MQTT broker). Clients can subscribe to these topics and receive whatever messages are published on those topics. It can support a variety of task and could be implemented quite easily with a low cost. The other benefits of MQTT are as follows.

Publish Subscribe model provides one-to-many message delivery, Uses TCP/IP for network connectivity ,Can work with SSL/TLS for security ,Very simple specification and APIs, making it easier for developers to work with MQTT based products, Most important APIs are CONNECT, PUBLISH, SUBSCRIBE, UNSUBSCRIBE, and DISCONNECT , The message header is short in MQTT and smallest packet size in 2 bytes, making it ideal for small and constrained devices

The best feature is this we can modify this technology according to other use, there are many software and technology that are present and support mqtt which make it more valuable with passage of time by increasing the use of MQTT Broker we can also increase the overall performance of the system however this system cannot work alone we need MQTT Broker/server for this Eclipse Mosquitto and Eclipse Paho is used Paho provides mqtt client libraries in multiple languages both publisher and subscriber while Eclipse Mosquitto is an open source mqtt broker server.. Based on the lightweight MQTT protocol, Mosquitto is ideal for devices, sensors and other 'Internet of Things' devices, with low processing capacity. MQTT clients can connect to a given 
Mosquitto broker and publish/ subscribe the messages from a topic. Eclipse Mosquitto's main responsibility is to provide a communication channel between publishers/senders and subscribers/ receiver. This all connected with the sensor while provide the operator full functionality to his devices after mqtt we can send data directly to our computation station and again at the time of result we can transmit data to MQTT this is how this system could be integrated and used this system in the industrial scale. There are many open source resources available for both technology and their post growth and potential estimates indicate that alone only their economic values will be in trillions.

The Proposed system will give some new capabilities to both of these technologies as ANN will learn thing faster than ever before and IoT will be more responsive also new functionality or traits will be easier to find out by these traits/Functionality our system can find any irregularity in data and can resolve that issue as early as possible better and efficient ways in the field of Security will evolve and we overall productivity of the system will increase because of this.

\section{APPLICATIONS}

In this section we describe a wide set of safety mechanism that could be developed and significantly improved by using this integrated style of technology Thanks to the increased computation power and the boom of the IoT sensor market and open source system which has make the third party use reliable cities like Barcelona are generating millions of dollar by using IoT technologies is their parking system also it help them to save energy and fuel this could bee significantly improved by using ANN as in times of any natural calamity and situation this system can react and notify it's customer about the danger also efficient use of traffic lights could be possible as ANN could detect the wastage of electricity by the data provided by IoT Moreover this system will prove to be helpful in the all the field were we can generate and record data.

Smart City-As an emerging Technology this can fit into all types of geolocations acquiring data from different infrastructure available in the city which can be studied by ANN and helped to develop new Mechanism for safety, Monetization and preservation of natural resources also it will give a better experience to the citizen as services can found the flaws in them by ANN and recover in time of any emergency we can save our infrastructure by the best possible option as it works on real time there will be a high probability of success with minimum loss making city more safe and productive.

Petrochemicals Industry- Petrochemical industry faces a great challenge of safety and economic loss due to accidents these things can be addressed by IoT and ANN as Petrochemical industry uses and produce many toxic chemical with the existing technology it is possible to sense this toxic chemical by IoT and with use of ANN we can draw real time conclusion and tack action to stop any disaster this could be easily integrated with the existing infrastructure as both the sensor and ANN technologies to recognize unwanted substances are available also it could help to check the quality of crude by checking Sulphur and other content making it beneficial for environment purpose as well.

Defense Technology-It will help to increase the safety of equipment's because of data provided by IoT and then Accessed by ANN we can find the limitations and weakness of military equipment's. at the time of war/Practice ANN can sense the danger and can help operator to perform it's task..
Thus it will help to construct new generation of weapon and unmanned weapon could be in practical use because ANN as it try to replicate the human brain. IoT Sensor will help us gather data about the machinery which can be very beneficial for future research. Intelligent management of resource, maximizing output, Reducing causalities are some of the benefits possible.

Resource Management- Self Adjusting Solar panel and other improvement to the existing technology is possible as ANN could find the most productive ways and that could be implemented in the real time like sun position is changed by 1 degree in every 4 minutes this affects the output of solar panel but with ANN we can overcome this problem as panel could adjust itself at best angle possible. Effiency of tidal energy can be increased by studying the behavior of tides by using IoT sensor and then transmitting that data to ANN. Other resources like mineral can also be benefited as sensor can detect chemical from which we can found which chemical is present their and by using ANN we reach the best possible ways to extract mineral.

Safety Measures. - there are many industries like mining and cement where the working conditions are vulnerable and chances of any calamity are high by using this technology we can study situation more effectively which will help to construct new safety standards and backup plan, it will also help to know the environment impact. Ex- Bhopal gas tragedy Like incident could be avoided which was reason of around 30000 deaths as we can sense unwanted chemical and other unwanted component it will help to detect malfunctions in initial stages ANN can detect different types of gases and substances by changing composition .and it's circumstances and be figured out. These industries generate large amount of data for IoT sensor which could also be used for making beneficial changes in the existing system.

Healthcare- This Technology enable cost effective, efficient, timely, and high-quality ubiquitous medical services. Pervasive healthcare applications generate a vast amount of sensor data that must be managed properly for further analysis and processing this sensor data when managed Through ANN while help to find latest ways to cure much faster then ever before. Things like Mitosis detection is possible by this technology and will help in the health care research field as well. It works on real time and at time of emergency doctor. It will make health communication more fast and effective due to sensor and ANN health monitoring will also increase it will help in complicated cases and ANN can predict the output.

Space Technology - Many exploration mission failed because of the unknown condition of universe ANN can help to make better estimate of things for human and help develop new generation technology also data taken from this mission will highly beneficial for research disaster like NASA Challenger mission can be completely saved as real time data and Computational power of ANN will help to detect the problem and gives accurate prediction on it and help to boost space technology moreover the design of various space technology equipment has been remain same for last 60 years with ANN breakthrough on new design is possible which will increase our space exploration capacity.

It can create high resolution simulation helps in making safety mechanism and design for building and industries ex Smoke Simulation of high resolution could be developed making whole system more predictable By ANN it could also create 3D model of objects by using 2D Training data set it create $3 \mathrm{~d}$ model hierarchy means step based. 
It also help in Intelligence purpose by using Audio Training set we can create Facial Animation these data set can be obtained on real time by IoT making this system really quick in real time. Thus we can go from text to speech and then to a virtual actor making it more friendly for human also.

Thus, best in class performance on problems that significantly outperform other solution in multiple domain could can be derived in a short span of time by increasing the profitability and decreasing the threat of the failure. It reduces the need of feature engineering which is the most time-consuming part of machine learning.

\section{CHALLENGES}

It requires a large amount of data if there is a small set of training data then ANN could give inappropriate result as it's totally based on the input. It requires very high computational power and is expensive to train the most complex model takes week to train using hundreds of subsystems equipped with GPUs. Determining the topology/flavor/training method/hyperparameters for deep learning is a black art with no theory to guide you. Incompleteness of the data set will also be a problem. The time taken for large scale deep learning is in Table 1.

\begin{tabular}{|c|c|c|c|}
\hline Methods & $\begin{array}{l}\text { Computing } \\
\text { Power }\end{array}$ & $\begin{array}{l}\text { Number of exam- } \\
\text { ples and free pa- } \\
\text { rameters }\end{array}$ & $\begin{array}{l}\text { Average } \\
\text { running } \\
\text { time }\end{array}$ \\
\hline DBN [41] & $\begin{array}{l}\text { NVIDIA GTX } \\
280 \text { GPU with } 1 \\
\text { GB mwmory }\end{array}$ & $\begin{array}{l}\text { One million imag- } \\
\text { es and } 100 \\
\text { minllion parame- } \\
\text { ters }\end{array}$ & $\sim 1$ day \\
\hline CNN [55] & $\begin{array}{lr}\text { Two GTX } & 580 \\
\text { GPUs, } & \text { each } \\
\text { with } & 3 \mathrm{~GB} \\
\text { memory } & \\
\end{array}$ & $\begin{array}{l}1.2 \text { million high } \\
\text { resolution ( } 256 \mathrm{x} \\
\text { 256) images and } 60 \\
\text { million parameters }\end{array}$ & $\tilde{\sim}^{5-6}{ }^{5-6}$ \\
\hline $\begin{array}{l}\text { DisBelief } \\
\text { [56] }\end{array}$ & $\begin{array}{lr}1,000 & \text { CPUs } \\
\text { with Downpour } \\
\text { SGD } & \text { with } \\
\text { Adagrad } & \\
\end{array}$ & $\begin{array}{l}1.1 \text { billion audio } \\
\text { examples and } 42 \\
\text { million model } \\
\text { parameters }\end{array}$ & $\begin{array}{l}\sim 16 \\
\text { hours }\end{array}$ \\
\hline $\begin{array}{l}\text { Sparse au- } \\
\text { toencoder } \\
\text { [50] }\end{array}$ & $\begin{array}{ll}1,000 & \text { CPUs } \\
\text { with } & 16,000 \\
\text { cores } & \\
\end{array}$ & $\begin{array}{l}10 \text { million } 200 \times \\
200 \text { pixel images } \\
\text { and one billion } \\
\text { parameters }\end{array}$ & $\sim 3$ days \\
\hline $\begin{array}{l}\text { COTS HPC } \\
\text { [58] }\end{array}$ & $\begin{array}{l}64 \text { NVIDIA } \\
\text { GTX } 680 \text { GPUs, } \\
\text { each with } 4 \text { GB } \\
\text { memory }\end{array}$ & $\begin{array}{l}10 \text { million } 200 \times \\
200 \text { images and } 11 \\
\text { billion parameters }\end{array}$ & -3 days \\
\hline
\end{tabular}

High volumes of data present a great challenging issue for deep learning. Big data often possesses many examples (inputs), large varieties of class types (outputs), and very high dimensionality (attributes).

These properties directly lead to running-time complexity and

model complexity. The sheer volume of data makes it often impossible to train a deep learning algorithm with a central processor and storage. Instead, distributed frameworks with parallelized machines are preferred. Recently, impressive progresses have been made to mitigate the challenges related to high volumes. The novel models utilize clusters of CPUs or GPUs in increasing the training speed without scarifying accuracy of deep learning algorithms. Strategies for data parallelism or model parallelism or both have been developed. For example, data and models are divided into blocks that fit with in-memory data; the forward and backward propagations can be implemented effectively in parallel although deep learning algorithms are not trivially parallel although deep learning algorithm are not trivial parallel. However, there is a need of another framework in future to scale significantly beyond current network. high performance computing infrastructure-based systems together with theoretically sound parallel learning algorithms or novel architectures.
Another challenge associated with high volumes is the data incompleteness and noisy labels. Unlike most conventional datasets used for machine learning, which were highly curated and noise free, Data Set is often incomplete resulting from their disparate origins. To make things even more complicated. Their need to be more advanced ANN algorithm to deal with this type of situation which may arise in complex system very often. However unsupervised dataset will also increase the accuracy of the trained ANN system. There are Emerging systems as well as there is no accurate defined protocol of follow for construction of this integrate system most of the test are on a experimental level and this technology is in it's growing phase.

A key challenge in the design of accelerators is to balance the trade-off between efficiency and flexibility i.e., the computing elements and memory hierarchy should be designed such that they retain a large part of the efficiency due of specialization, while still providing significant programmability. As an example, in order to characterize this trade-off space in the context of Artificial learning networks, we hierarchically breakdown the computations present in both the testing and training phases.

There are many significant challenges on Integrating IoT Security is one of the major issue it could be divided into three Parts Authorization, Open ports and Encryption other issues are battery consumption, CPU consumption, Low bandwidth issue. There is also issue that 48 -bit Ipv4 address become too small for worldwide IoT.

However, some of this issue can be easily deal with mqtt and other technologies.

Privacy and security challenge is also concerned a threat as the system is contains data sets which could be wrong or anybody can play wrongfully from these data. For this we need good security system to make entire system closed from the outside disturbance.

Standard Challenges Include as the entire system needs to be constructed differently as different project needs different data sets which need to be deal differently.

\section{CONCLUSION}

Hence I conclude this model that by integrating ANN and IoT we can Easily help eliminate human error and change different things that are not possible manually we will be able to develop new safety guidelines for many things which is really helpful

Industries like IoT is expected to be widely applied .For example, the food industry is integrating WSN and RFID to build automated systems for tracking, monitoring, and tracing food quality along the food supply chain in order to improve food quality and other industries like that work on Fluid which faces a problem of corrosion and damage of this can be deal with the help of this system we can generate data useful for the protection and getting insights about the health of all the products in the industry. It will help corporations to understand data more easily which will help to change their trends according to market. It also discusses how certain technology components, i.e., Eclipse Mosquito and Eclipse Paho can be used to implement this system these protocol has already been used in IoT and is reliable. By whole implementation in various field as stated by the paper it can achieve the goal of intelligent identifying, locating, tracking, monitoring, and managing things. However, there will be challenges in the field in terms of complexity and privacy challenges. 


\section{REFERENCES}

[1] Internet of Things in Industries: A Survey Li Da Xu, Senior Member, IEEE, Wu He, and Shancang Li

[2] Internet of Things Feng Xia, Laurence T. Yang, Lizhe Wang and Alexey Vinel

[3] Artificial Intelligence and Big Data Daniel E. O'Leary, University of Southern California

[4] Smart Objects as Building Blocks for the Internet of Things Frédéric Thiesse+

[5] Efficient Embedded Learning for IoT Devices Swagath Venkataramani, Kaushik Roy and Anand Raghunathan School of Electrical and Computer Engineering, Purdue University

[6] Internet of things : A vision, architectural Elements, and future Directions Jayavardhana Gubbi , Rajkumar Buyya , Slaven Marusic ,Marimuthu palaniswami
[7] Deep Learning: Methods and Applications Li dong, Don yu

[8] Challenges in Deep Learning Plamen Angelov1 and Alessandro Sperduti

[9] A systemic approach for IoT security Arbia Riahi*, Yacine Challal †, Enrico Natalizio $\dagger$, Zied Chtourou*, Abdelmadjid Bouabdallah

[10] Deep Learning-Based Classification of Hyperspectral Data Yushi Chen, Zhouhan Lin, Xing Zhao, Gang Wang, Member, Yanfeng Gu

[11] DeepX: A Software Accelerator for Low-Power Deep Learning Inference on Mobile Devices Nicholas D. Lane, Sourav Bhattacharya, Petko Georgiev Claudio Forlivesi, Lei Jiao, Lorena Qendro*, and Fahim Kawsar 